ARTICLE

Received 24 Jun 2014 | Accepted 12 Sep 2014 | Published 27 Oct $2014 \quad$ DOl: 10.1038/ncomms6264

\title{
Mitochondrial defects trigger proliferation of neighbouring cells via a senescence-associated secretory phenotype in Drosophila
}

Mai Nakamura ${ }^{1,2, \star}$, Shizue Ohsawa ${ }^{1, \star} \&$ Tatsushi Igaki ${ }^{1,3}$

Cell-cell interactions play important roles in epithelial tumorigenesis. Here we show in Drosophila imaginal epithelium that Ras activation and mitochondrial dysfunction, frequent alterations in cancers, cause cellular senescence and senescence-associated secretory phenotype (SASP), which leads to overgrowth of neighbouring tissue. Ras-activated cells express several hallmarks of cellular senescence such as elevation of senescence-associated $\beta$-galactosidase activity, upregulation of the Cdk inhibitor Dacapo, heterochromatinization and cellular hypertrophy. Strikingly, defects in mitochondrial function cause Ras-activated cells to undergo DNA damage response, cell cycle arrest and thereby induce SASP, exhibiting full aspects of cellular senescence. Mechanistically, mitochondrial defects in conjunction with Ras cause production of reactive oxygen species, downregulation of CycE activity and activation of $\mathrm{p} 53$, which cooperate together to trigger a cell cycle arrest-Jun $\mathrm{N}$-terminal kinase (JNK) feedback loop that amplifies JNK activation, leading to upregulation of the inflammatory cytokine Unpaired. Our data suggest that mitochondrial defects promote Ras-induced cellular senescence and thereby contribute to tumour progression through SASP.

\footnotetext{
${ }^{1}$ Laboratory of Genetics, Graduate School of Biostudies, Kyoto University, Yoshida-Konoecho-cho, Kyoto 606-8501, Japan. ${ }^{2}$ Division of Genetics, Kobe University Graduate School of Medicine, 7-5-1 Kusunoki-cho, Kobe 650-0017, Japan. ${ }^{3}$ PRESTO, Japan Science and Technology Agency (JST), 4-1-8 Honcho, Kawaguchi 332-0012, Japan. ${ }^{\star}$ These authors contributed equally to this work. Correspondence and requests for materials should be addressed to T.I. (email: igaki@lif.kyoto-u.ac.jp).
} 
C ell-cell interactions within tumour microenvironment play a crucial role in epithelial cancer development. For instance, communication among oncogenic cells, stromal fibroblasts, immune cells and normal epithelial cells can drive tumour development and progression ${ }^{1,2}$. Although the mechanism by which oncogenic cells cooperate with surrounding cells to drive tumour progression is still elusive, recent studies have suggested that cellular senescence, the state of ultimate and irreversible cell cycle arrest, can promote cancer development through cell-cell communication. Cellular senescence is provoked by diverse cellular stresses such as chromosomal instability, telomere shortening, oxidative stress, DNA damage and aberrant activation of oncogenes ${ }^{3-6}$. The oncogene-induced cellular senescence has been shown to exert a tumour-suppressive effect by inducing cell cycle arrest in premalignant cells ${ }^{3,4,6}$. In fact, premalignant human nevi and colon adenomas contain cells exhibiting cellular senescence phenotypes, but senescent cells are markedly reduced in malignant melanomas and adenocarcinomas developed from these lesions ${ }^{7,8}$. In addition, in mouse models with activated Ras signalling or pten deletion, senescent cells are abundant in premalignant lesions but are scarce in cancers eventually developed ${ }^{9-11}$. These observations raise a question of how tumours eventually emerge from such heterogeneous oncogenic lesions with a significant population of senescent cells. Interestingly, recent studies in cell culture systems have revealed non-autonomous functions of senescent cells; cells undergoing cellular senescence secrete a variety of cytokines, chemokines and growth factors that affect surrounding cells, which is known as the senescence-associated secretory phenotype (SASP) or the senescence-messaging secretome $e^{4,6,12-16}$. Thus, in certain settings, cells exhibiting SASP could exert a tumourpromoting effect on their surrounding cells ${ }^{15,17}$. For instance, senescent fibroblasts secrete an epidermal growth factor-like growth factor amphiregulin and a cytokine growth-related oncogene- $\alpha$, both stimulate proliferation of premalignant epithelial cells in cell culture systems ${ }^{12,18}$. In addition, some SASP factors such as interleukin-6 (IL-6) and IL-8 can promote malignant transformation in conjunction with activated oncogenes $^{19-21}$. Furthermore, it has been shown in obesity mouse model that SASP factors play crucial roles in promoting development of obesity-associated hepatocellular carcinoma ${ }^{22}$. However, the molecular link between cellular senescence phenotypes and SASP, as well as the mechanism by which cellular senescence drives SASP induction in vivo, are poorly understood.

Drosophila provides a powerful model system for studying cell-cell communication within developing epithelium through the genetic mosaic technique ${ }^{23}$. We have recently performed a genetic screen in Drosophila imaginal epithelium to identify genes that drive tumour progression through cell-cell interactions and found that mutations in mitochondrial respiratory complexes in Ras-activated cells ( Ras $^{\mathrm{V} 12} / \mathrm{mito}^{-/}$cells) induce tumour progression in their neighbouring benign tumours ${ }^{24}$. Ras ${ }^{\mathrm{V} 12} /$ mito $^{-1-}$ cells upregulate production of an inflammatory cytokine Unpaired (Upd, an IL-6 homologue) and thereby promote growth and malignancy of surrounding Ras-induced benign tumours, exhibiting marked similarity with SASP.

Here we show in Drosophila imaginal epithelium that clones of cells activated Ras signalling express several markers of cellular senescence such as elevation of senescence-associated $\beta$-galactosidase (SA- $\beta$-gal) activity, upregulation of G1 cyclin-dependent kinase (Cdk) inhibitor Dacapo (Dap, a p21/p27 homologue), heterochromatinization and increased cell size (cellular hypertrophy). Intriguingly, despite the upregulated expression of Dap, Ras-activated cells do not undergo cell cycle arrest. We find that mitochondrial dysfunction causes Ras ${ }^{12}$-expressing cells to induce cell cycle arrest in G1 phase, thereby leading to SASP. Our data suggest that mitochondrial defect causes Ras-activated cells to undergo cellular senescence in vivo. Furthermore, we dissect the genetic pathway of cellular senescence-mediated tumour progression through SASP.

\section{Results}

Ras induces cellular senescence markers in Drosophila. Aberrant activation of oncogenes such as Ras can induce cellular senescence in mammalian cells. However, it has been unclear whether the oncogene-induced cellular senescence can also be induced in invertebrates. We first examined whether aberrant activation of Ras signalling in Drosophila imaginal epithelium causes cellular senescence. We found that clones of cells expressing oncogenic Ras ${ }^{\mathrm{V} 12}$ in the eye imaginal disc indeed exhibited several cellular senescence markers such as elevated SA- $\beta$-gal activity (Fig. 1a,b), upregulation of a Cdk inhibitor Dacapo (Dap, a p21/p27 homologue; Fig. 1c,d, Supplementary Fig. 1a,b), elevation of histone $\mathrm{H} 3-\mathrm{K} 9$ trimethylation (which is associated with the senescence-associated heterochromatin foci; Fig. 1e,f) and upregulation of heterochromatin protein-1 (HP-1; which binds to methylated K9 of histone H3; Supplementary Fig. 1c,d). In mammalian cell culture systems, cells undergoing cellular senescence exhibit enlarged and flattened cell morphology. Similarly, we found that cells activating Ras signalling in imaginal epithelium showed increased cell size $(1.20 \pm 0.02$, relative to wild-type cells of $1.00 \pm 0.02$ ) (Fig. $1 \mathrm{~g}, \mathrm{~h}$ ). Thus, cells with elevated Ras signalling in Drosophila epithelium exhibit several hallmarks of cellular senescence.

Mitochondrial defect causes Ras ${ }^{\mathrm{V} 12}$ cells to induce $\mathrm{G} 1$ arrest. Cells undergoing cellular senescence exhibit an irreversible cell cycle arrest in G1 phase, which is one of the critical cellular events in cellular senescence. We thus examined whether clones of Ras-activated cells cause cell cycle arrest in G1 phase in imaginal disc using the cell cycle monitoring probe S/G2/M-Green ${ }^{25,26}$. Despite showing several phenotypic markers of cellular senescence (Fig. 1), Ras ${ }^{\mathrm{V} 12}$-expressing cells were not arrested in G1 phase, as most of these cells did not lose S/G2/M-Green expression (Fig. 2a,b, quantified in Fig. 2e). These results indicate that Ras activation is not sufficient for inducing all aspects of cellular senescence phenotypes and therefore Ras ${ }^{\mathrm{V} 12}$-expressing cells are not senescent cells. These observations are consistent with previous reports in mammalian systems that SA- $\beta$-gal, increased cell size and senescence-associated heterochromatin foci are neither necessary nor sufficient for induction of cellular senescence ${ }^{27-30}$. Intriguingly, we found that, when mitochondrial respiratory function was simultaneously downregulated in Ras ${ }^{\mathrm{V} 12}$-expressing cells (by introducing the mitochondrial respiratory complex I mutation $P d s w^{-/-}$), such mutant cells caused cell cycle arrest in G1 phase (Fig. 2d, quantified in Fig. 2e), whereas cells with mitochondrial dysfunction alone only slightly suppressed cell cycle progression (Fig. 2c, quantified in Fig. 2e). Similarly, by introducing the $P d s w^{-/}$mutation or the mitochondrial respiratory complex IV mutation $\mathrm{CoVa}^{-/-}$ (hereafter, $\mathrm{Pds} w^{-/-}$and $\mathrm{CoVa}^{-/-}$are referred to as mito ${ }^{-/-}$), we have recently found that mitochondrial dysfunction in $\mathrm{Ras}^{\mathrm{V} 12}$-expressing cells (Ras ${ }^{\mathrm{V} 12} /$ mito $^{-/}$- cells) exhibit SASPlike phenotype, as such mutant cells upregulate production of the IL-6 homologue Upd, although cells expressing Ras ${ }^{\mathrm{V} 12}$ alone do not $^{24}$. In addition, Ras ${ }^{\mathrm{V} 12} /$ mito $^{-/-}$cells, but not Ras ${ }^{\mathrm{V} 12}$ cells or mito $^{-/}$- cells, exhibited intranuclear foci of phosphorylated histone $\mathrm{H} 2 \mathrm{~A}$ variant $(\gamma \mathrm{H} 2 \mathrm{Av}$; Fig. $2 \mathrm{f}-\mathrm{j})$, an indicator of DNA damage response that plays a crucial role in the induction of cellular senescence ${ }^{31-34}$ and SASP ${ }^{35,36}$. Furthermore, 
Wild type
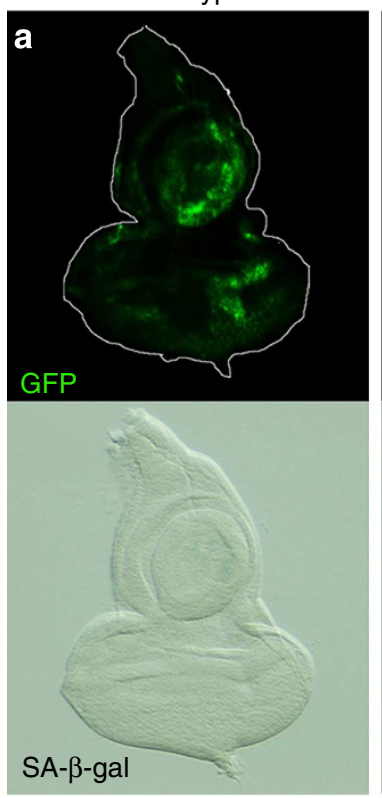

Wild type
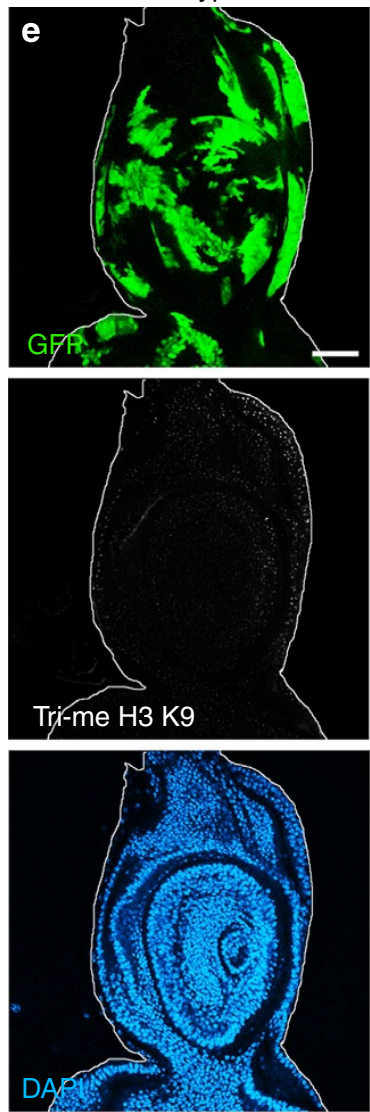

$\operatorname{Ras}^{\mathrm{V} 12}$

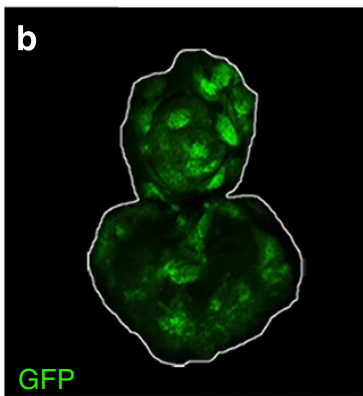

GFP

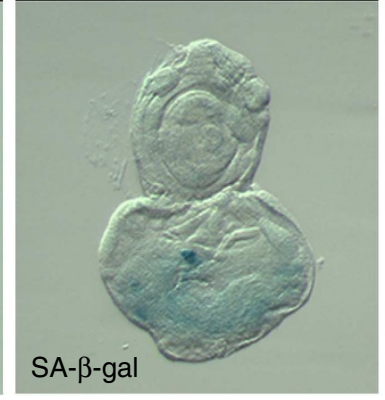

Ras $^{\mathrm{V} 12}$
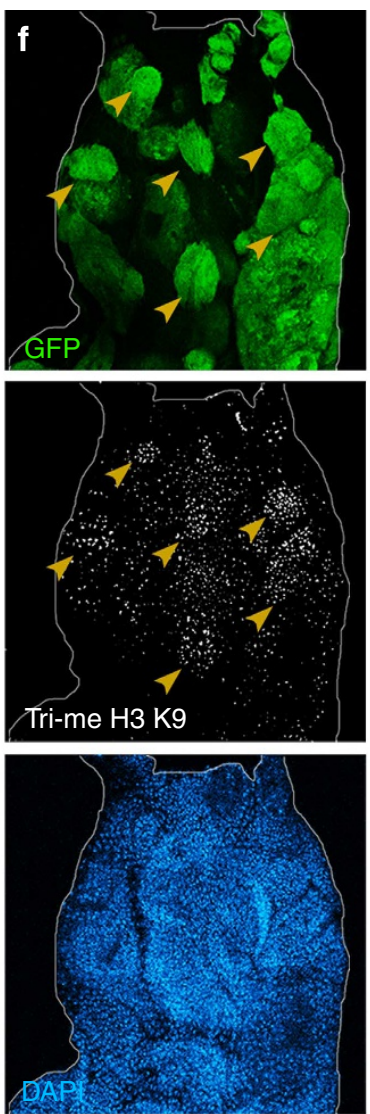

Wild type
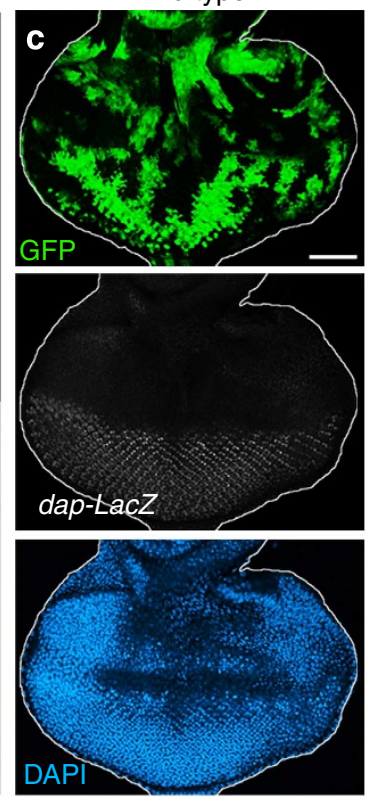

Wild type
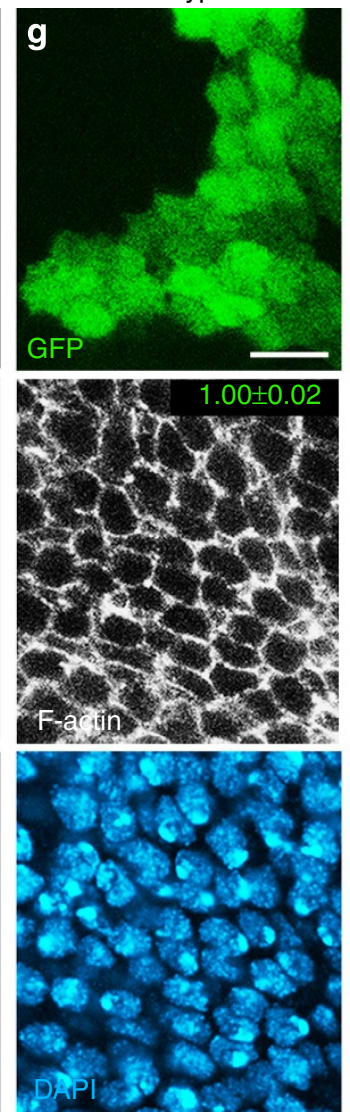

$\operatorname{Ras}^{\mathrm{V} 12}$
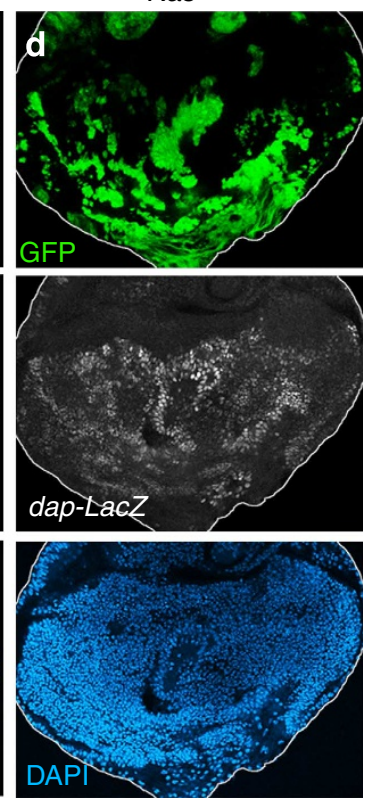

$\operatorname{Ras}^{\mathrm{V} 12}$
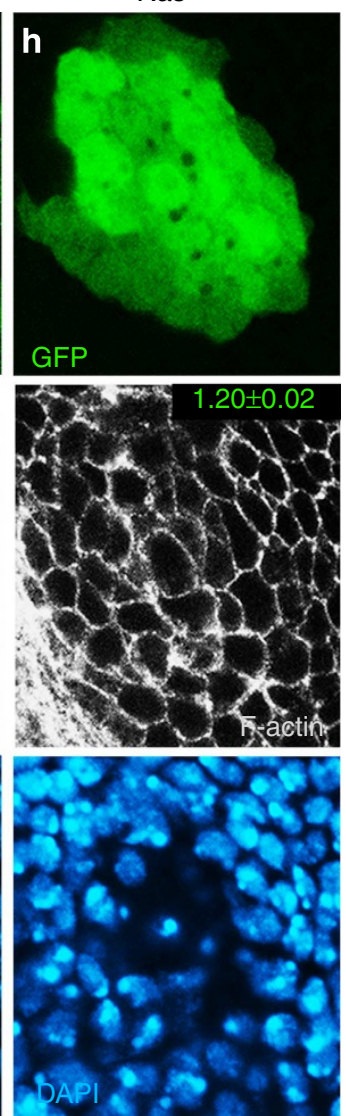

Figure 1 | Ras activation induces cellular senescence markers in Drosophila. (a,b) Eye-antennal disc bearing green fluorescent protein (GFP)-labelled wild-type (a) or Ras V12 (b) clones was subjected to SA- $\beta$-gal assay. (c,d) GFP-labelled wild-type (c) or Ras V12 (d) clones were induced in dap-LacZ eye-antennal disc and stained with anti- $\beta$-gal. (e,f) Eye-antennal disc bearing GFP-labelled wild-type (e) or Ras ${ }^{\mathrm{V} 12}$ (f) clones was stained with anti-Histone-H3-trimethyl-K9. Arrowheads indicate representative clones upregulating trimethylated Histone H3-K9. (g,h) Eye-antennal disc bearing GFP-labelled wild-type $(\mathbf{g})$ or Ras ${ }^{\mathrm{V} 12}$ (h) clones was stained with phalloidin to visualize F-actin. Cell size was measured using ImageJ and was quantified as relative size to wild-type cells. Data were collected as mean \pm s.e., wild-type $(n=186)$; $\operatorname{Ras}^{\mathrm{V} 12}(n=227)$. The nuclei were stained with 4',6-diamidino-2-phenylindole (DAPI). Scale bars, $50 \mu \mathrm{m}(\mathbf{c}, \mathbf{e})$ or $10 \mu \mathrm{m}(\mathbf{g})$. See Supplementary Table 1 for genotypes. 
mitochondrial defect strongly enhanced cellular hypertrophy in $\mathrm{Ras}^{\mathrm{V} 12}$-expressing cells $(1.93 \pm 0.03$, relative to wild-type cells of $1.00 \pm 0.02$; Fig. $2 \mathrm{l}$ ), whereas mitochondrial dysfunction alone did not affect cell size $(0.97 \pm 0.01$, relative to wild-type cells of
$1.00 \pm 0.02$; Fig. $2 \mathrm{k}$ ). These results indicate that mitochondrial dysfunction causes Ras-activated cells to induce cellular senescence. Indeed, $\mathrm{Ras}^{\mathrm{V} 12} / \mathrm{mito}^{-/-}$cells showed all other characters of cellular senescence observed in $\mathrm{Ras}^{\mathrm{V} 12}$-expressing
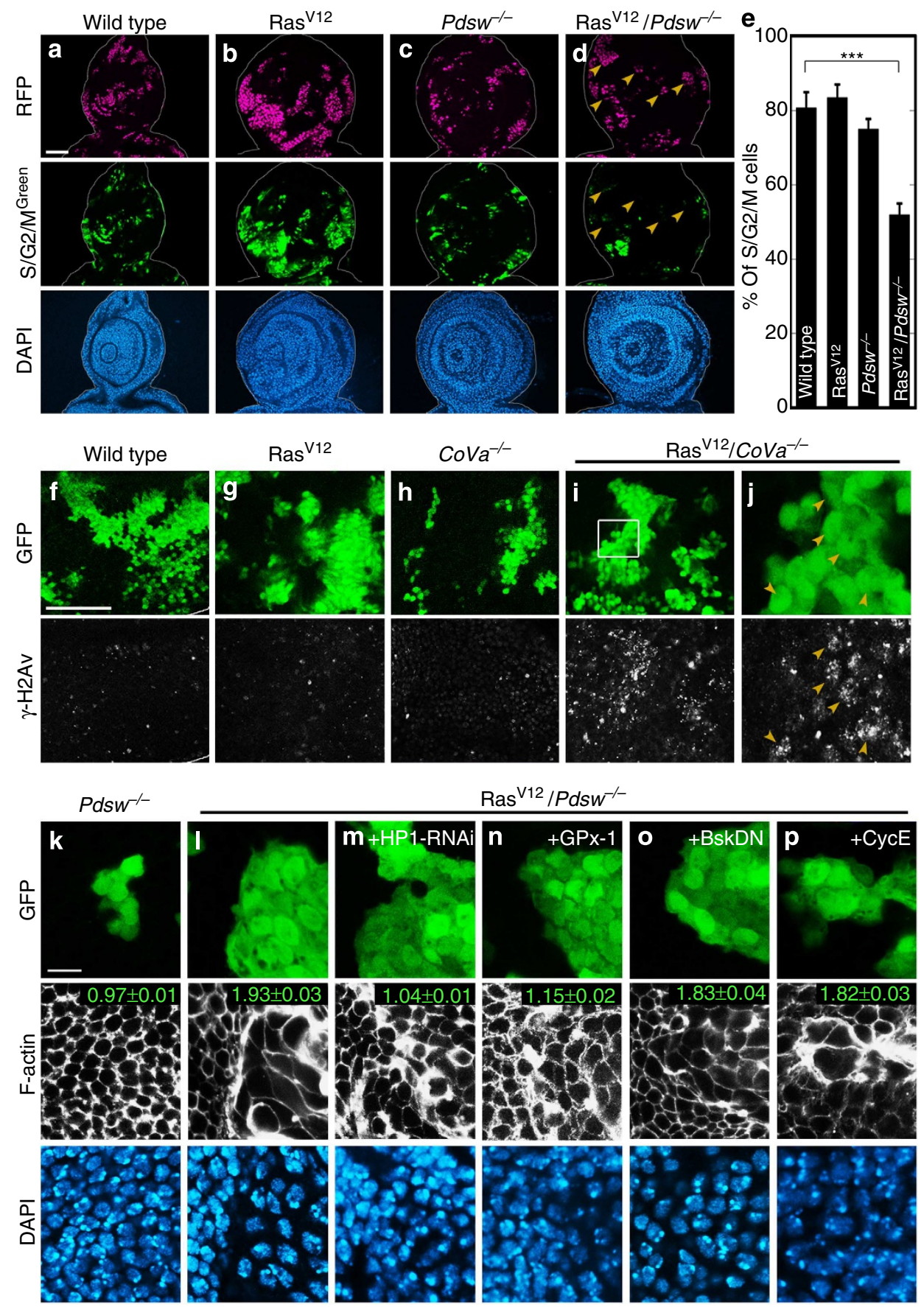

q

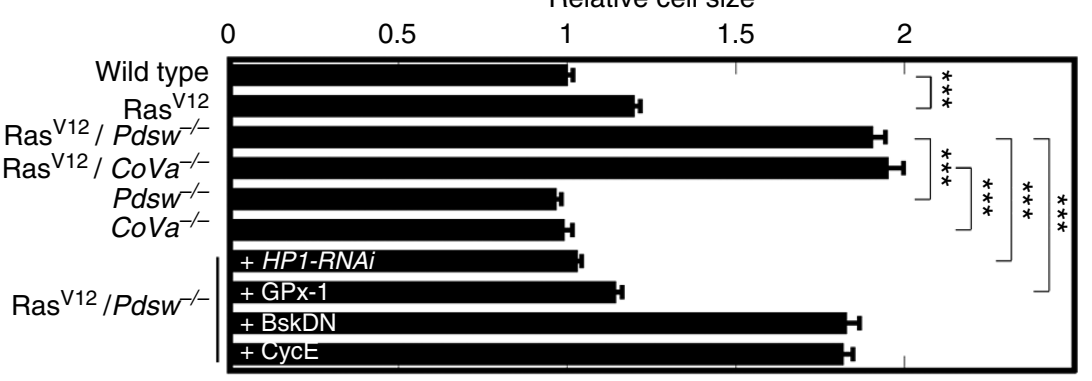


cells such as SA- $\beta$-gal activity, upregulation of $\mathrm{p} 21 / \mathrm{p} 27 / \mathrm{Dap}$, histone $\mathrm{H} 3-\mathrm{K} 9$ trimethylation and upregulation of $\mathrm{HP}-1$, whereas mitochondrial dysfunction alone did not induce such changes (Supplementary Fig. 2a-n).

SASP and cellular hypertrophy are separable processes. Although there have been several studies on the mechanism by which senescent cells induce SASP factors, it is still unclear how the cellular senescence phenotypes are linked to SASP induction. We therefore sought to identify the molecules that link cellular senescence markers to SASP. When HP-1 expression was reduced by HP-1-RNAi in $\mathrm{Ras}^{\mathrm{V} 12} /$ mito $^{-/-}$clones, cellular hypertrophy was almost completely abolished, placing cellular hypertrophy downstream of heterochromatin formation (Fig. $2 \mathrm{~m}$, quantified in Fig. 2q). However, blocking of both HP-1 expression and cellular hypertrophy did not suppress induction of non-autonomous overgrowth (Supplementary Fig. 2o, quantified in Supplementary Fig. 2p), suggesting that SASP and cellular hypertrophy are separable processes in cellular senescence. It has been shown that $\mathrm{Ras}^{\mathrm{V} 12} / \mathrm{mito}^{-/}$cells upregulate Upd through production of reactive oxygen species (ROS) and subsequent Jun N-terminal kinase (JNK) activation ${ }^{24}$. We found that blocking oxidative stress by overexpression of glutathione peroxidase (GPx-1) in $\mathrm{Ras}^{\mathrm{V} 12} /$ mito $^{-}{ }^{-}$clones significantly suppressed cellular hypertrophy, whereas blocking JNK signalling by Bsk ${ }^{\mathrm{DN}}$ did not affect the hypertrophy (Fig. 2n,o, quantified in Fig. 2q). Importantly, expression of either GPx-1 or $\mathrm{Bsk}^{\mathrm{DN}}$ in Ras $\mathrm{V}^{\mathrm{V} 2}$ / mito $^{-/-}$clones abolished non-autonomous overgrowth ${ }^{24}$. These data indicate that two cellular senescence markers, heterochromatinization and oxidative stress, are required for cellular hypertrophy, but the cellular hypertrophy is not essential for SASP induction.

G1 cell cycle arrest is required for SASP. We then sought to identify other cellular senescence markers responsible for SASP induction. Our genetic data so far presented indicate that mitochondrial dysfunction causes Ras-activated cells to undergo cellular senescence by inducing G1 arrest and SASP, suggesting the possible molecular link between cell cycle arrest and SASP induction. Strikingly, overexpression of CycE, which counters the function of p21/p27/Dap and promotes the G1/S transition, in $\mathrm{Ras}^{\mathrm{V} 12} / \mathrm{mito}^{-/}$clones significantly suppressed non-autonomous overgrowth (Fig. 3a,b, Supplementary Fig. 3a-c, quantified in Supplementary Fig. 3g,h). Furthermore, overexpression of CycE blocked induction of upd expression (Fig. 3c, compare with Supplementary Fig. 3d) as well as its upstream JNK activation (visualized by the puc-lac $Z$ reporter ${ }^{37,38}$; Fig. 3d, compare with Supplementary Fig. 3e), both of which are essential for the induction of non-autonomous overgrowth by $\mathrm{Ras}^{\mathrm{V} 12} /$ mito $^{-/-}$ clones $^{24}$. In contrast, overexpression of CycE had no effect on the induction of oxidative stress (as visualized by the gstD-GFP reporter ${ }^{39}$; Fig. 3e, compare with Supplementary Fig. 3f), which acts upstream of JNK activation in this signalling ${ }^{24}$, as well as the induction of cellular hypertrophy (Fig. 2p, quantified in Fig. 2q). These data suggest that cell cycle arrest is required for SASP induction at the downstream of oxidative stress. Indeed, we found that forced induction of G1 arrest by loss of $c y c E$ causes $\mathrm{Ras}^{\mathrm{V} 12}$-expressing clones to induce non-autonomous overgrowth (Fig. 3f), whereas clones with $c y c E$ mutation alone do not (Fig. 3g). Similar to the case of Ras ${ }^{V 12} / m_{i t o}{ }^{-1-}$ clones, the nonautonomous overgrowth caused by $\mathrm{Ras}^{\mathrm{V} 12} / \mathrm{cycE}^{-/-}$clones was suppressed by blocking JNK signalling (Fig. $3 \mathrm{~h}$, quantified in Supplementary Fig. 3i) or Yki activity (Fig. 3i, quantified in Supplementary Fig. 3j), a transcriptional co-activator that induces upd expression in $\mathrm{Ras}^{\mathrm{V} 12} /$ mito $^{-1}-$ clones $^{24}$. Indeed, Ras $\mathrm{V} 12 /$ $c y c E^{-/-}$clones exhibited JNK activation (Fig. 3l) and Upd induction (Fig. $3 \mathrm{n}$ ), whereas Ras ${ }^{\mathrm{V} 12}$ alone or $c y c E$ mutation alone caused neither of them (Fig. $3 \mathrm{k}, \mathrm{m}$, and data not shown) ${ }^{24}$. We also found that a reduction in $R b$ gene, a cell cycle inhibitor that is negatively regulated by $\mathrm{CycE}$, significantly suppressed nonautonomous overgrowth caused by $\mathrm{Ras}^{\mathrm{V} 12} /$ mito $^{-/}$clones (Fig. 3j, quantified in Supplementary Fig. 3k). Together, these data indicate that cell cycle arrest in G1 phase causes Rasactivated cells to induce SASP.

Cell cycle arrest amplifies JNK signalling. The fact that overexpression of CycE blocks JNK activation in $\mathrm{Ras}^{\mathrm{V} 12} /$ mito $^{-/-}$ cells raised the possibility that JNK signalling is activated through cell cycle arrest. To examine this possibility, we first utilized a JNK-dependent cell death phenotype caused by overexpression of the tumour-necrosis factor ligand Eiger. Ectopic expression of Eiger in the posterior region of the eye disc by the GMR-Gal4 driver causes JNK-dependent cell death that leads to a small eye phenotype $^{40}$ (Fig. 4a). Significantly, co-expression of CycE suppressed the small eye phenotype induced by Eiger (Fig. 4b). Conversely, when the expression of $\mathrm{CycE}$ was reduced by $c y c E$ RNAi, the small eye phenotype was significantly enhanced (Fig. 4c). Importantly, overexpression of $\mathrm{CycE}$ or $c y c E-\mathrm{RNAi}$ did not affect significantly the eye morphology (Fig. $4 \mathrm{~d}-\mathrm{f}$ ). We also found that although overexpression of Eiger or $c y c E$-RNAi in undifferentiated eye disc cells by the eyeless-Gal4 driver did not affect eye morphology (Fig. 4g,h), co-expression of Eiger and cycE-RNAi in these cells caused small eye phenotype (Fig. 4i), which was cancelled by blocking JNK signalling (Fig. 4j). These results suggest that CycE negatively regulates JNK signalling activity. We thus directly confirmed this using the JNK kinase Hemipterous (Hep). Overexpression of a constitutively active form of Hep (Hep $\left.{ }^{C A}\right)$ in the posterior region of the eye disc led to the activation of JNK in this area (Fig. 4k). Significantly, co-expression of CycE suppressed this JNK activation (Fig. 4l), confirming that CycE antagonizes JNK activation. These data

Figure 2 | Mitochondrial defect causes Ras $\mathbf{V 1 2}$ cells to undergo G1 arrest and cellular hypertrophy. (a-d) Eye-antennal disc bearing RFP-labelled wildtype (a), $\operatorname{Ras}^{\mathrm{V} 12}(\mathbf{b}), P d s w^{-/-}$(c) or Ras ${ }^{\mathrm{V} 12} / \mathrm{Pdsw}^{-/-}$(d) clones was induced in S/G2/M-Green eye-antennal disc. Arrowheads indicate representative clones lacking S/G2/M-Green expression. (e) Quantification of \% of S/G2/M-Green-positive cells in each clone. Five to eight imaginal discs were examined using ImageJ. S/G2/M-Green-positive cells were significantly fewer in Ras ${ }^{12} / P d s w^{-/}-$clones. Data were collected as mean \pm s.e., wild type $(n=5) ; \operatorname{Ras}^{V 12}(n=5) ; P d s w^{-/-}(n=8) ; \operatorname{Ras}^{V 12} / P d s w^{-/-}(n=5)$. Student's $t$-test, ${ }^{\star \star \star} P<0.0001$. (f-j) Eye-antennal disc bearing green fluorescent protein (GFP)-labelled wild-type (f), Ras ${ }^{\mathrm{V} 12}(\mathbf{g}), \mathrm{CoVa}^{-/-}(\mathbf{h})$ or Ras ${ }^{\mathrm{V} 12} / \mathrm{CoVa}^{-/-}(\mathbf{i}, \mathbf{j})$ clones was stained with anti- $\gamma-\mathrm{H} 2 \mathrm{Av}$. (j) High-magnification image of the boxed area in $\mathbf{i}$. Arrowheads in indicate cells exhibiting typical intranuclear $\gamma-\mathrm{H} 2 \mathrm{Av}$ foci. (k-p) Eye-antennal disc bearing GFP-labelled

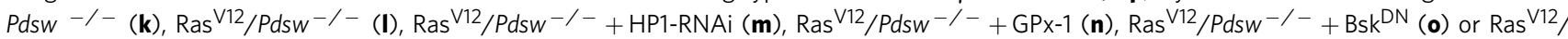
$P d s w^{-/-}+$CycE (p) clones was stained with phalloidin. (q) Cell size was measured using ImageJ and was quantified as relative size to wild-type cells. Data were collected as mean \pm s.e., wild-type $(n=186) ; \operatorname{Ras}^{V 12}(n=227) ; \operatorname{Ras}^{V 12} / P d s w^{-/-}(n=339) ; \operatorname{Ras}^{V 12} / \mathrm{CoVa}^{-/-}(n=194) ; P d s w^{-/-}(n=103)$; $\mathrm{CoVa}^{-/-}(n=99) ; \mathrm{Ras}^{\mathrm{V} 12} / \mathrm{Pdsw}^{-/-}+\mathrm{HP1} 1 \mathrm{RNAi}(n=335) ; \mathrm{Ras}^{\mathrm{V} 12} / \mathrm{Pdsw}^{-/-}+\mathrm{GPx}-1(n=167) ; \mathrm{Ras}^{\mathrm{V} 12} / \mathrm{Pdsw}^{-/-}+\mathrm{Bsk}^{\mathrm{DN}}(n=188) ; \mathrm{Ras}^{\mathrm{V} 12} /$ $P d s w^{-/-}+$CycE $(n=180)$. Student's $t$-test, ${ }^{\star \star \star} P<0.0001$. The nuclei were stained with $4^{\prime}, 6$-diamidino-2-phenylindole (DAPI). Scale bars, $50 \mu \mathrm{m}$ (a,f) or $10 \mu \mathrm{m}$ (k). See Supplementary Table 1 for genotypes. 

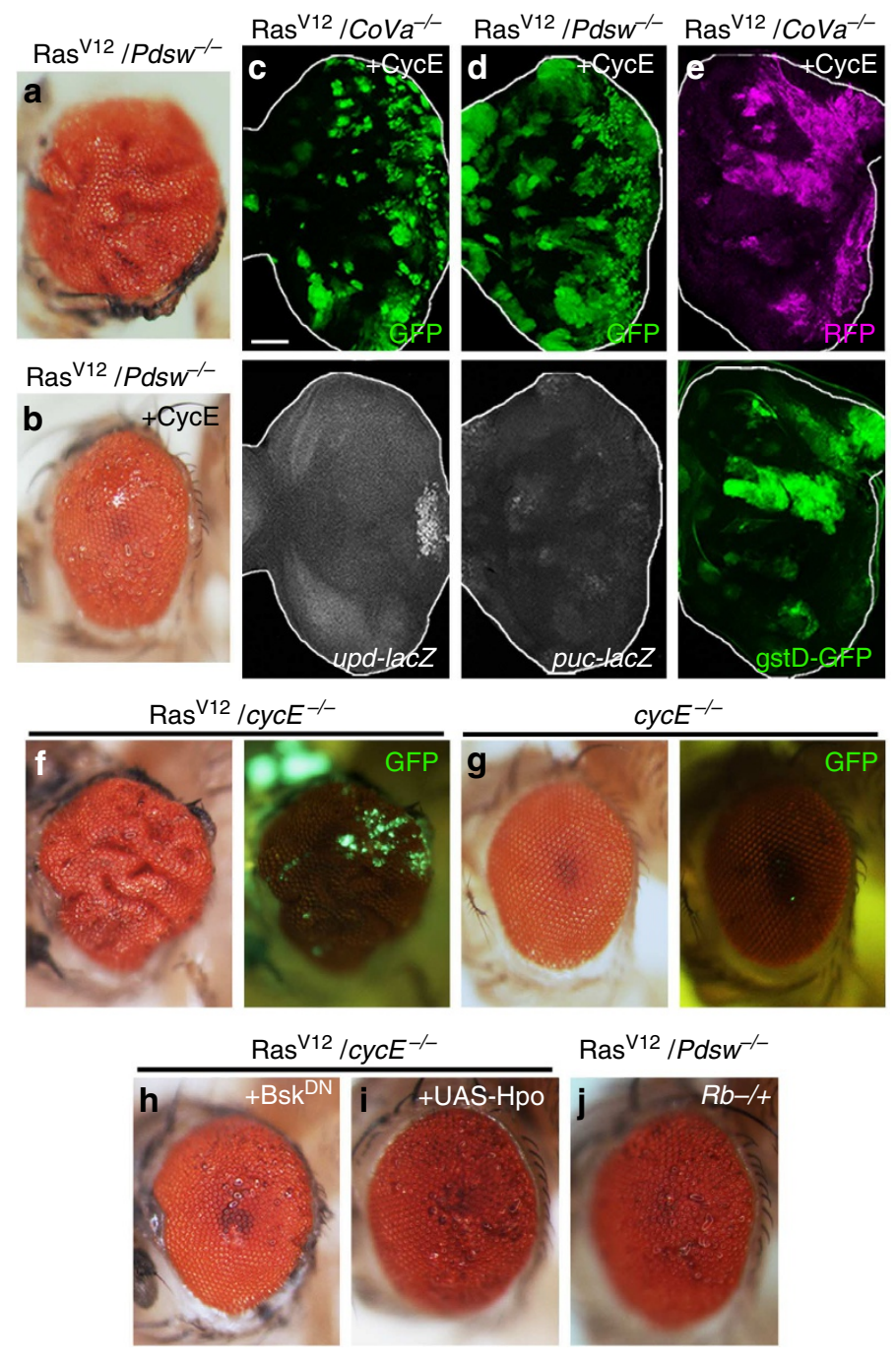

$\operatorname{Ras}^{\mathrm{V} 12} / \mathrm{Pdsw} w^{-/}$

$c y c E^{-/-}$
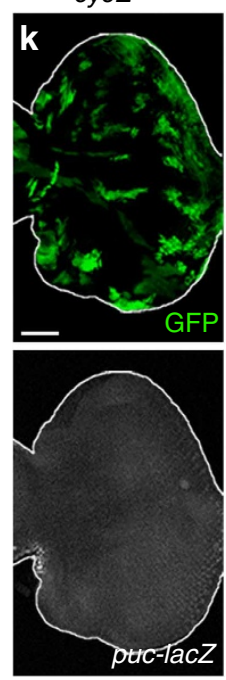
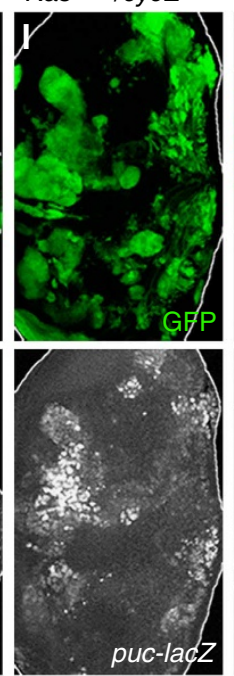

cycE $E^{-/-}$
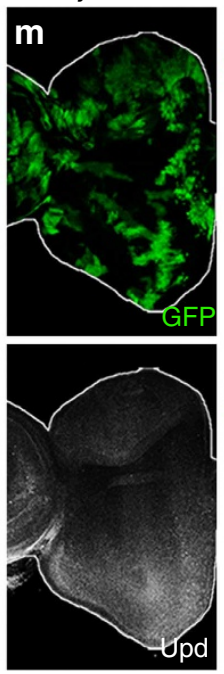

$\operatorname{Ras}^{\mathrm{V} 12} / \mathrm{CycE}^{-/-}$

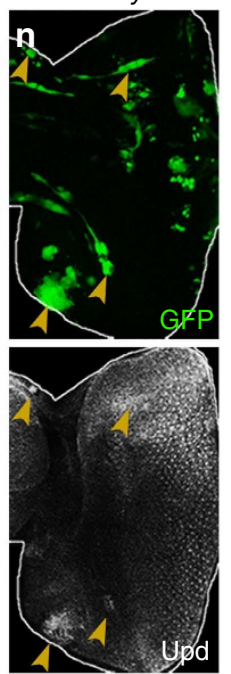

Figure 3 | Cell cycle arrest is required for inducing SASP. (a,b) Adult eye bearing Ras $\mathrm{V} 12 / P d s w^{-/-}$(a) or Ras $\mathrm{V} 12 / P d s w-/-+C y c E(\mathbf{b}) c l o n e s$. See Supplementary Fig. 3a for wild-type eye. (c) Green fluorescent protein (GFP)-labelled Ras V12/CoVa- ${ }^{-}-$CycE clones were induced in upd-LacZ

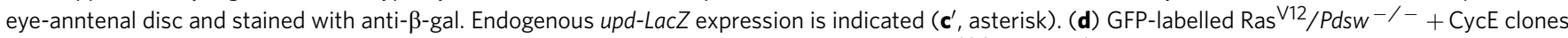
were induced in puc-LacZ eye-anntenal disc and stained with anti- $\beta$-gal. (e) RFP-labelled Ras $\mathrm{V}^{2} / \mathrm{CoVa}^{-} /-$+ CycE clones were induced in gstD-GFP

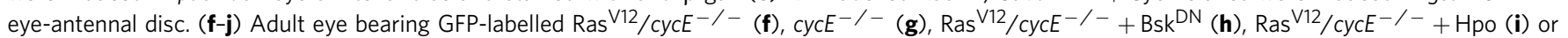

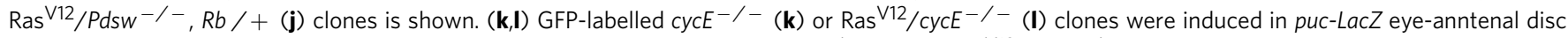
and stained with anti- $\beta$-gal. ( $\mathbf{m}, \mathbf{n})$ Eye-antennal disc bearing GFP-labelled $c y c E^{-/-}(\mathbf{m})$ or Ras $\mathrm{V} 12 / c y c E^{-/-}$(n) clones was stained with anti-Upd. Arrowheads indicate representative clones upregulating Upd. Scale bars, $50 \mu \mathrm{m}$. See Supplementary Table 1 for genotypes. 
GMR > Eiger
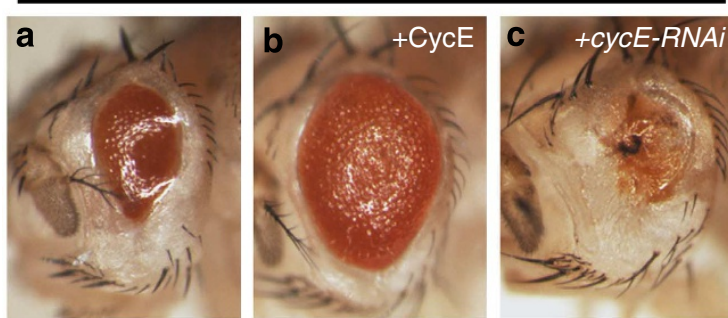

GMR-Gal4
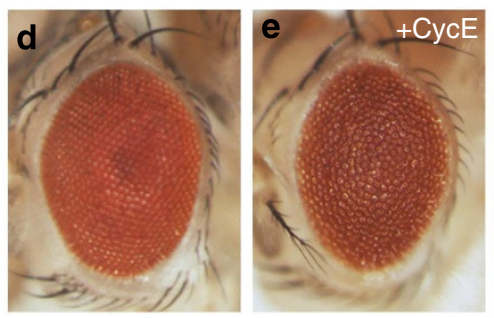

GMR $>\mathrm{Hep}^{\mathrm{CA}}$
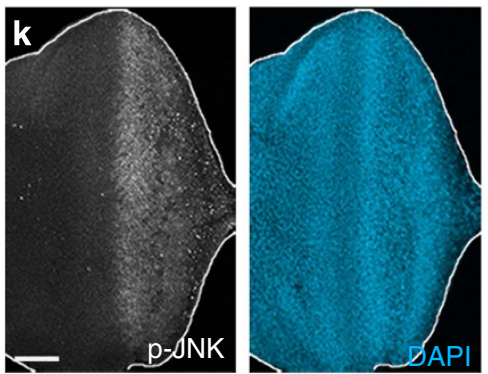

GMR $>\mathrm{Hep}^{\mathrm{CA}}$
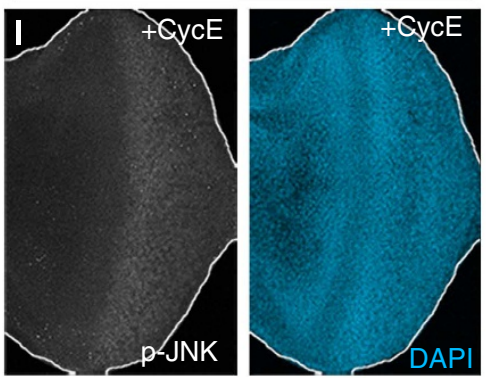

o

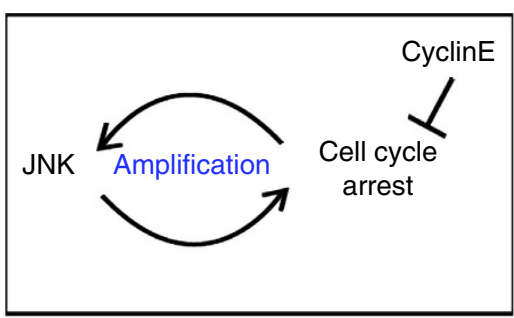

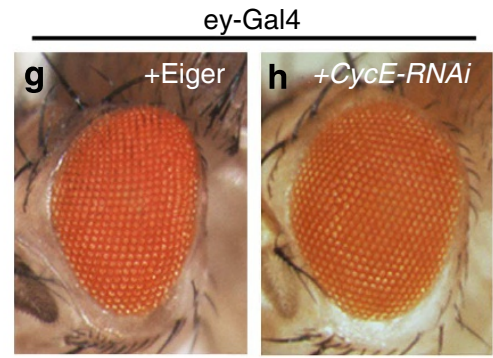

ey-Eiger, $c y c E-R N A i$
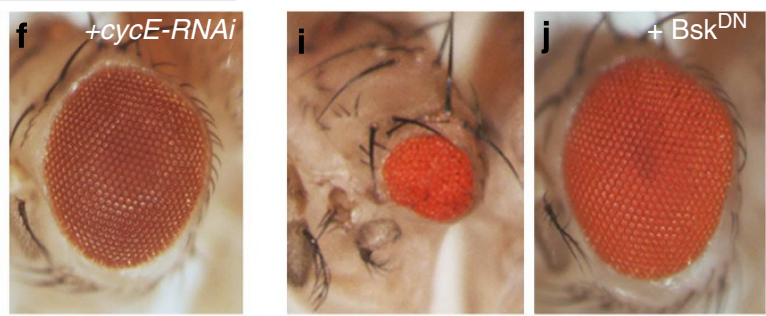

Wild type
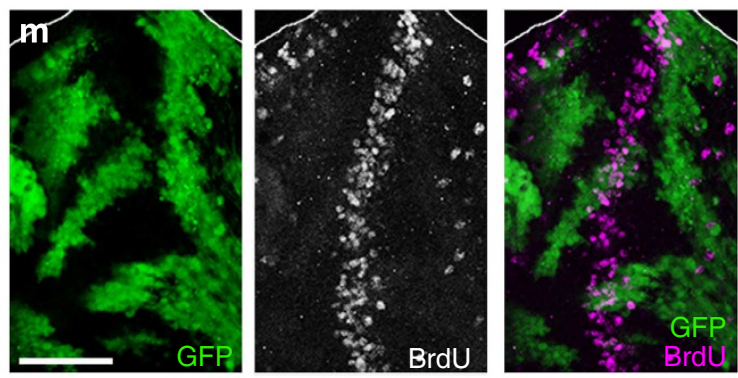

$\mathrm{Hep}^{\mathrm{CA}}+\mathrm{p} 35$
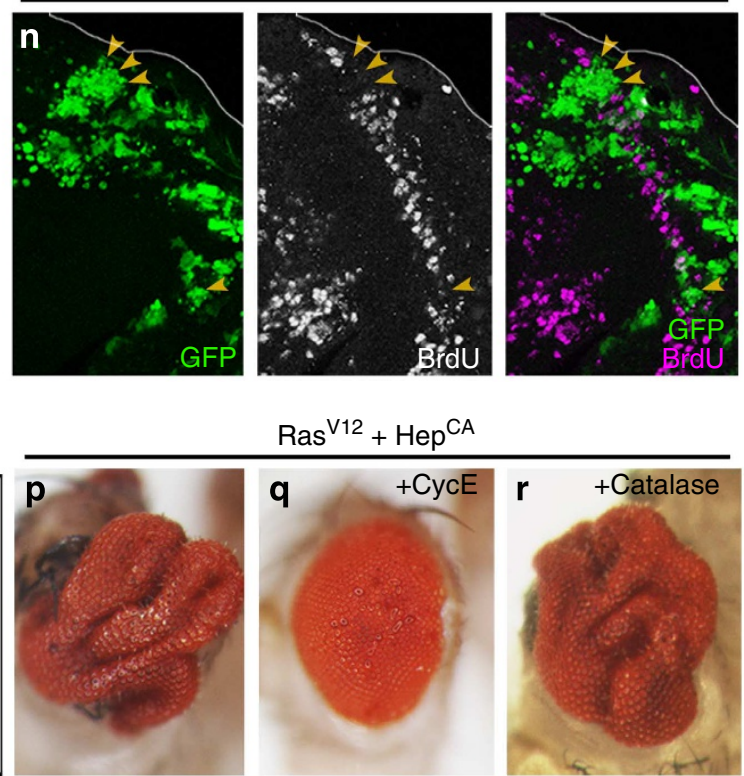

Figure 4 | Cell cycle arrest amplifies JNK activation. (a-j) Eye phenotypes of GMR $>$ Eiger (a), GMR $>$ Eiger + CycE (b), GMR $>$ Eiger $+c y c E-R N A i(\mathbf{c})$,

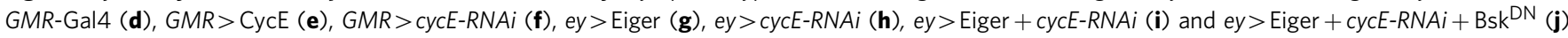
are shown. (k,I) Eye disc expressing $\operatorname{Hep}^{C A}(\mathbf{k})$ or HepCA + CycE (I) in the posterior region (right half) was stained with anti-phospho-JNK antibodies. The nuclei were stained with 4',6-diamidino-2-phenylindole (DAPI). ( $\mathbf{m}, \mathbf{n}$ ) 5-Bromodeoxyuridine (BrdU) labelling in eye disc bearing green fluorescent protein (GFP)-labelled wild-type (m) or Hep ${ }^{\mathrm{CA}}+\mathrm{p} 35$ (n) clones is shown. Arrowheads indicate second mitotic wave (SMW) cells lacking BdrU staining. (o) A model for the cell cycle arrest-JNK amplification loop in Ras $\mathrm{V} 12 /$ mito $^{-/-}$senescent cells. (p,q,r) Adult eye bearing Ras ${ }^{\mathrm{V} 12}+\mathrm{Hep}^{\mathrm{CA}}$ (p), $\mathrm{Ras}^{\mathrm{V} 12}+\mathrm{Hep}^{\mathrm{CA}}+\mathrm{CycE}(\mathbf{q})$ or Ras ${ }^{\mathrm{V} 12}+\mathrm{Hep}^{\mathrm{CA}}+$ Catalase $(\mathbf{r})$ clones is shown. Scale bars, $50 \mu \mathrm{m}$. See Supplementary Table 1 for genotypes. 

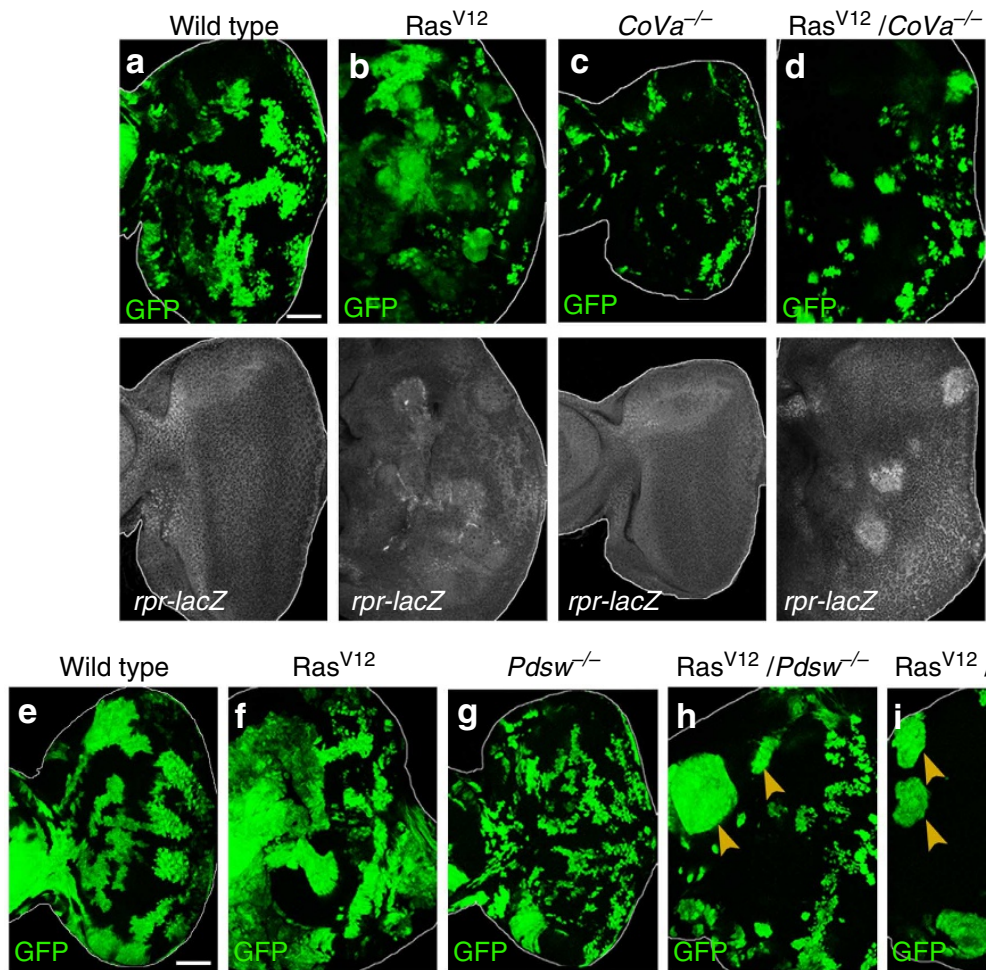

$\operatorname{Ras}^{\mathrm{V} 12}$

$\mathrm{Pdsw}^{-1-}$
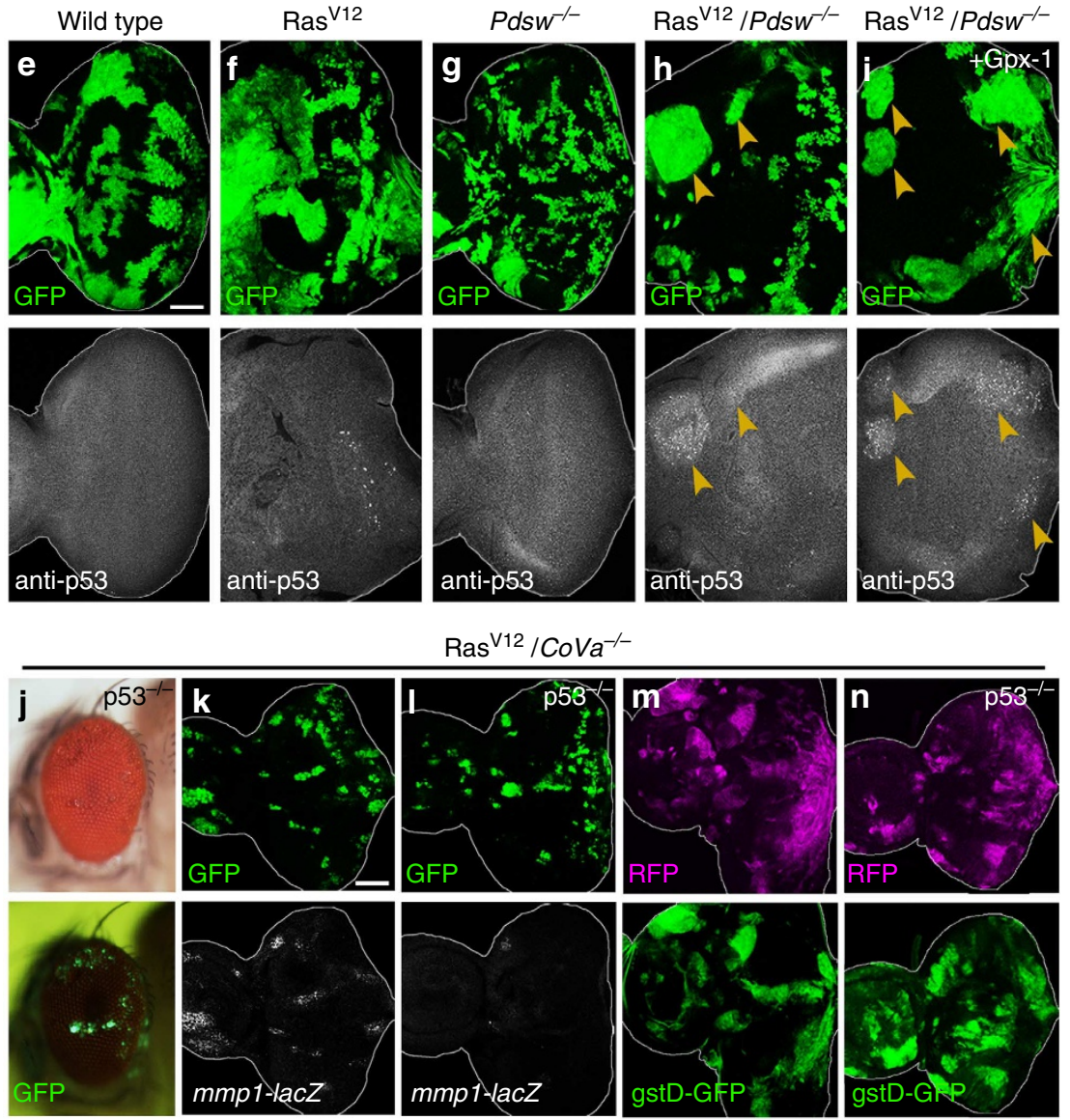

$\mathrm{Ras}^{\mathrm{V} 12} / \mathrm{CoVa}^{-/}$
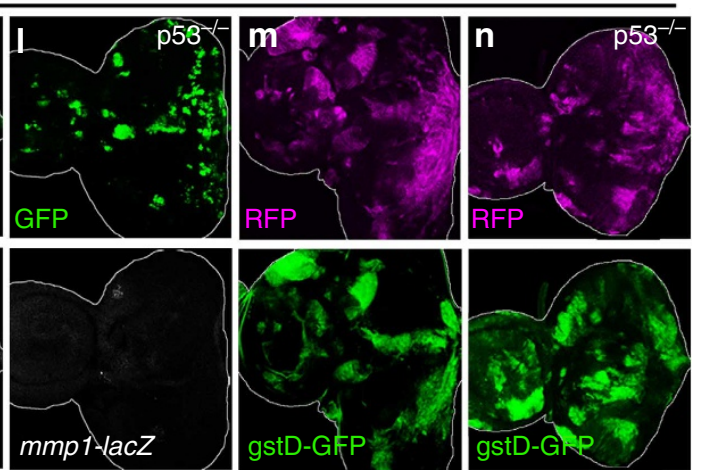

$\operatorname{Ras}^{\mathrm{V} 12}+\mathrm{p} 53$

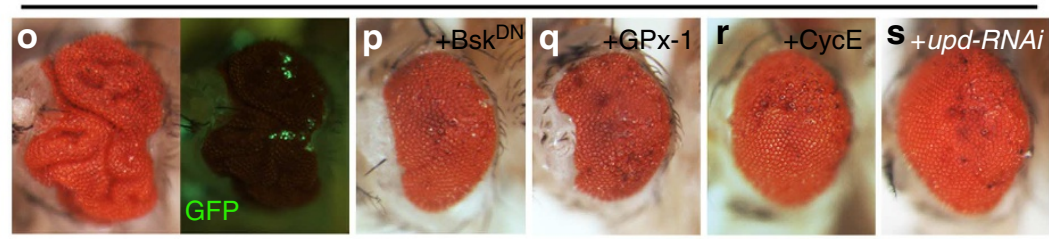

Figure 5 I p53 mediates cell cycle arrest-JNK-dependent SASP. (a-d) Green fluorescent protein (GFP)-labelled wild-type (a), Ras ${ }^{\mathrm{V} 12}$ (b), CoVa-/(c) or Ras ${ }^{\mathrm{V} 12} / \mathrm{CoVa}^{-/-}$(d) clones were induced in rpr-lacZ eye-antennal disc and stained with anti- $\beta$-gal. (e-i) Eye-antennal disc bearing GFP-labelled wild-type (e), $\operatorname{Ras}^{\mathrm{V} 12}(\mathbf{f}), \mathrm{Pdsw}^{-/-}(\mathbf{g}), \mathrm{Ras}^{\mathrm{V} 12} / \mathrm{Pdsw}{ }^{-/-}$(h) or Ras $\mathrm{V12} / \mathrm{Pdsw}{ }^{-/-}+\mathrm{GPx}-1$ (i) clones was stained with anti-p53 antibody. Arrowheads indicate representative clones upregulating p53. (j) Adult eye bearing GFP-labelled Ras ${ }^{\mathrm{V} 12} / \mathrm{CoVa}^{-/-}, d_{m p 53^{-/-}}$clones. (k,I) GFP-labelled Ras $\mathrm{V12} /$ $\mathrm{CoVa}^{-/-}(\mathbf{k})$ or Ras $\mathrm{V}^{2} / \mathrm{CoVa}^{-/-}, \mathrm{dmp} 53^{-/-}$(I) clones were induced in mmp1-lacZ eye-antennal disc and stained with anti- $\beta$-gal. (m,n) RFP-labelled $\mathrm{Ras}^{\mathrm{V} 12} / \mathrm{CoVa}^{-/-}(\mathbf{m})$ or Ras $\mathrm{V} 12 / \mathrm{CoVa}^{-/-}, \mathrm{dmp}^{-} 3^{-/-}(\mathbf{n})$ clones were induced in gstD-GFP eye-antennal disc. (o-s) Adult eye bearing Ras $\mathrm{V} 12+\mathrm{p} 53$ (o), $\mathrm{Ras}^{\mathrm{V} 12}+\mathrm{dmp} 3+\mathrm{Bsk}^{\mathrm{DN}}(\mathbf{p})$, Ras ${ }^{\mathrm{V} 12}+\mathrm{dmp} 53+\mathrm{GPx}-1(\mathbf{q})$, Ras ${ }^{12}+\mathrm{dmp} 53+\mathrm{CycE}(\mathbf{r})$ or Ras ${ }^{\mathrm{V} 12}+\mathrm{dmp} 53+$ upd-RNAi (s) clones is shown. Scale bars, $50 \mu \mathrm{m}$. See Supplementary Table 1 for genotypes. 


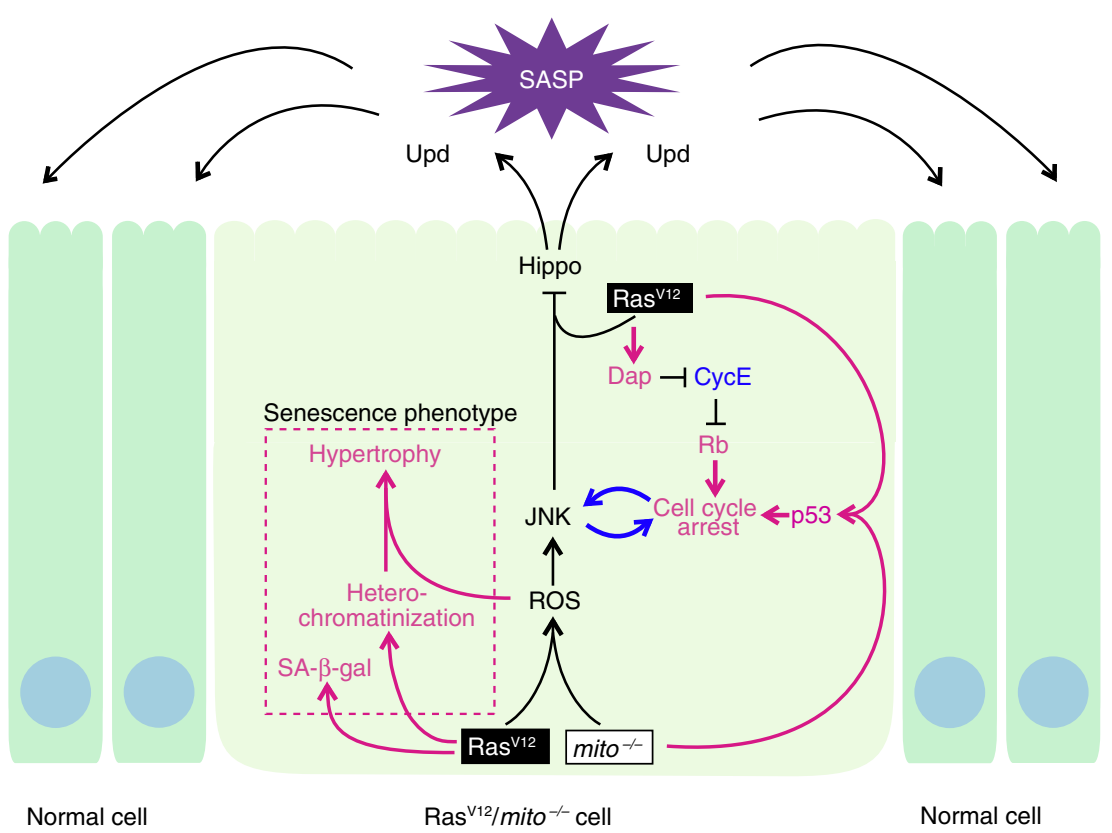

Figure 6 | A model for the genetic pathway to SASP by Ras activation and mitochondrial dysfunction. Simultaneous Ras activation and mitochondrial dysfunction lead to production of ROS, downregulation of CycE activity and activation of p53, which form the cell cycle arrest-JNK amplification loop. Activated JNK further cooperates with Ras signalling to inactivate the Hippo pathway, leading to upregulation of an inflammatory cytokine Upd. Our data show that mitochondrial defect promotes Ras-induced cellular senescence and thereby contributes to tumour progression of neighbouring tissue through SASP.

suggest that cell cycle arrest enhances JNK activation. Intriguingly, we found that JNK activation also induces cell cycle arrest, as clones of cells expressing Hep ${ }^{\mathrm{CA}}$ abolished BrdU incorporation in the second mitotic wave in the eye disc (Fig. $4 \mathrm{~m}, \mathrm{n}$ ), suggesting that cell cycle arrest and JNK activation forms a positive feedback loop that amplifies the signalling (Fig. 4o). To verify this model, we induced clones of cells co-expressing Ras ${ }^{12}$ and $\mathrm{Hep}^{\mathrm{CA}}$ in the eye disc, which have been shown to cause non-autonomous overgrowth ${ }^{41}$ (Fig. 4p). Strikingly, non-autonomous overgrowth caused by $\mathrm{Ras}^{\mathrm{V} 12}+\mathrm{Hep}^{\mathrm{CA}}$ clones was strongly suppressed by co-expression of CycE (Fig. 4q, quantified in Supplementary Fig. 4a), but not by co-expression of antioxidant enzyme Catalase (Fig. 4r, quantified in Supplementary Fig. 4b), consistent with our findings that cell cycle arrest acts downstream of oxidative stress and upstream of JNK activation. Together, these data show that cells undergoing cellular senescence induce SASP through cell cycle arrestmediated amplification of JNK signalling.

p53 mediates cell cycle arrest-JNK-dependent SASP. Finally, we sought to identify the molecular link between $\mathrm{Ras}^{\mathrm{V} 12} /$ mito $^{-} /-$ and cell cycle arrest. It has been shown in mouse fibroblasts that the tumour-suppressor gene p53 is required for inducing cell cycle arrest in cells undergoing Ras-induced cellular senescence ${ }^{42-44}$. In addition, it has been shown in Drosophila imaginal disc that p53 and JNK form a positive feedback loop that amplifies their activity/expression ${ }^{45}$. Interestingly, we found that $\mathrm{Ras}^{\mathrm{V} 12} /$ mito $^{-/}$- clones strongly upregulate a p53 target gene reaper (rpr; Fig. 5a,d), whereas Ras activation alone or mitochondrial dysfunction alone causes only a moderate or no induction of $r p r$ expression, respectively (Fig. 5b,c). Furthermore, $\mathrm{Ras}^{\mathrm{V} 12} /$ mito $^{-/}$- clones upregulated p53 expression level (Fig. 5e,h), whereas Ras ${ }^{\mathrm{V} 12}$ alone or mitochondrial mutation alone caused only a slight upregulation or no upregulation of p53 expression level, respectively (Fig. 5f,g). These results indicate that Ras activation and mitochondrial dysfunction cooperate to upregulate p53 activity and raise the possibility that cell cycle arrest in $\mathrm{Ras}^{\mathrm{V} 12} /$ mito $^{-/}$- cells is mediated by $\mathrm{p} 53$, as previously shown in $\mathrm{CoVa}^{-/-}$clones (cells mutant for mitochondrial respiratory complex IV) ${ }^{46}$. Indeed, removal of p53 in Ras ${ }^{\mathrm{V} 12}$ / mito $^{-/-}$clones significantly suppressed JNK activation (visualized by the mmp1-lacZ reporter ${ }^{47}$; Fig. 5l, compare with Fig. 5k) as well as non-autonomous overgrowth (Fig. 5j, quantified in Supplementary Fig. 5h). In contrast, removal of p53 did not suppress oxidative stress in $\mathrm{Ras}^{\mathrm{V} 12} /$ mito $^{-/}-$clones (Fig. 5n, compare with Fig. $5 \mathrm{~m}$ ). In addition, blocking oxidative stress did not suppress upregulation of $\mathrm{p} 53$ in $\mathrm{Ras}^{\mathrm{V} 12} /$ mito $^{-/-}$ clones (Fig. 5i), placing p53 in parallel to oxidative stress. These data suggest that elevated p53 activity in $\mathrm{Ras}^{\mathrm{V} 12} /$ mito $^{-/}-$cells triggers cell cycle arrest-mediated amplification of JNK signalling, which cooperates with Ras signalling to induce SASP. To test this possibility, we induced clones of cells overexpressing both p53 and Ras V12 in the eye disc and found that these clones indeed induce non-autonomous overgrowth (Fig. 5o). Furthermore, the non-autonomous overgrowth caused by Ras ${ }^{\mathrm{V} 12}+\mathrm{p} 53$ clones was significantly suppressed by JNK inhibition (Fig. 5p, quantified in Supplementary Fig. 5i), antioxidant GPx-1 expression (Fig. 5q, quantified in Supplementary Fig. 5j), CycE overexpression (Fig. 5r, quantified in Supplementary Fig. 5k) and Upd downregulation (Fig. 5s, quantified in Supplementary Fig. 5l), suggesting that the overgrowth phenotype was due to SASP. We further confirmed this by immunostaining analyses, which showed that $\mathrm{Ras}^{\mathrm{V} 12}+\mathrm{p} 53$ clones upregulated JNK activity as well as upd expression (Supplementary Fig. 5a,c,d,f), whereas clones overexpressing p53 alone caused neither JNK/upd upregulation (Supplementary Fig. 5b,e) nor non-autonomous overgrowth (Supplementary Fig. 5g). Altogether, our data indicate that cells with Ras activation and mitochondrial dysfunction lead to production of ROS, downregulation of CycE activity and activation of $\mathrm{p} 53$, all of which cooperate together to trigger cell cycle arrest-mediated amplification of JNK signalling and subsequent SASP induction (Fig. 6). 


\section{Discussion}

Although accumulating evidence has suggested that cell-cell interactions between premalignant cells and senescent cells within tumour microenvironment play important roles in cancer development, the mechanisms underlying such tumour progression have been elusive. In this study, we found in Drosophila imaginal epithelium that Ras activation and mitochondrial dysfunction cooperate to induce cellular senescence, which induces oncogenic SASP through the cell cycle arrest-JNK amplification loop. Our data unveil the genetic pathway of cellular senescence phenotypes such as heterochromatinization, cellular hypertrophy, cell cycle arrest and SASP (Fig. 6). Given that $\mathrm{Ras}^{\mathrm{V} 12} /$ mito $^{-1}-$ cells are resistant to apoptosis ${ }^{24}$, such senescent cells could exist in tumour tissue for a long period and contribute to tumour progression through chronic 'oncogenic inflammation' by SASP factors.

In mammalian systems, cellular senescence is caused through p53-mediated cell cycle arrest, which is induced by activation of oncogenes or other cellular stresses such as oxidative stress. Interestingly, we found in Drosophila epithelium that mitochondrial defects in Ras-activated cells trigger ROS production and p53 activation, both of which cooperate to trigger cell cycle arrestmediated amplification of JNK signalling, a key regulator for SASP induction. Although our data show that p53 is required for the onset of SASP, it has been reported in mammalian systems that p53 is dispensable for SASP or senescence-associated inflammatory response induction ${ }^{17,48}$. This discrepancy could be due to the differences in the triggers of cellular senescence (mitochondrial dysfunction, X-irradiation/replicative exhaustion or loss of casein kinase $1 \propto 1$ ) and/or cellular contexts (in vivo epithelium, cell cultures or organoid cultures) examined in the studies. Our data suggest that in in vivo situations cellular senescence and SASP are triggered or enhanced by additional cellular stresses such as mitochondrial dysfunction in conjunction with Ras activation. Given that mitochondrial respiratory function is frequently downregulated in many types of cancers ${ }^{49}$, similar mechanism of SASP induction could be involved in the progression of Ras-activated human cancers.

Studies in mammalian systems have shown that cells undergoing cellular senescence produce SASP factors through nuclear factor- $\kappa \mathrm{B}$ signalling or epigenetic control of SASP genes ${ }^{36,50,51}$. Intriguingly, our genetic study in Drosophila revealed that SASP factors are produced through inactivation of the Hippo pathway, a signalling often deregulated in human cancers ${ }^{52}$. A similar mechanism of SASP induction via the Hippo pathway may also be involved in mammalian tumour tissues with senescent cells. Indeed, a study in mice has shown that the SASP factor IL-6 is significantly upregulated in the liver doubly mutant for $m s t 1 / m s t 2$ (ref. ${ }^{53}$, mammalian orthologues of the hippo gene.

Our finding that the cellular senescence machinery also exits in invertebrates suggests that cellular senescence plays a role not only in tumour regulation but also in some evolutionarily conserved physiological contexts such as normal development. Interestingly, it has recently been reported that cellular senescence is required for tissue remodelling during mouse development ${ }^{54}$. Given that Ras signalling, a major trigger for cellular senescence, plays crucial roles in cell proliferation, cell survival, cell differentiation and tissue morphogenesis in both vertebrates and invertebrates, further genetic studies using the Drosophila model may unveil the physiological roles of cellular senescence during normal development and homeostasis.

\section{Methods}

Fly strains and generation of clones. Fluorescently labelled mitotic clones 23,55 were produced in larval imaginal discs using the following strains: $\mathrm{y}, \mathrm{w}$, eyFLP1; Act $>y^{+}>$Gal4, UAS-GFP; FRT82B, Tub-Gal80 (82B tester-1), eyFLP5,
Act $>y^{+}>$Gal4, UAS-GFP; FRT82B, Tub-Gal80 (82B tester-2), y, w, eyFLP1; Act $>y^{+}>$Gal4, UAS-mmRFP; FRT82B, Tub-Gal80 (82B RFP-tester), Tub-Gal80, FRT40A, UAS-Ras ${ }^{V 12}$; eyFLP6, Act $>y^{+}>$Gal4, UAS-GFP (40A Ras tester), Tub-Gal80, FRT40A; eyFLP6, Act $>y^{+}>$Gal4, UAS-GFP (40A tester). Additional strains used were as follows: UAS-Bsk ${ }^{\mathrm{DN}}$ (a gift from T. Adachi-Yamada), UAS-Hep, UAS-Hep ${ }^{C A}$ (a gift from T. Adachi-Yamada), UAS-Eiger, CoVa ${ }^{\text {tend }}$, $d m p 53^{[11-1 B-1]}$ (a gift from U. Banerjee), mmpl-lacZ (a gift from M. Uhlirova), rpr-11-lacZ (a gift from J. Abrams), upd-lacZ (a gift from H. Sun), UAS-S/G2/ M-Green fucci ${ }^{26}$, UAS-GPx-1 (a gift from F. Missirlis), UAS-CycE, UAS-p35, UAS-dmp53, gstD-GFP (a gift from D. Bormann), Pdsw $w^{k 10101}, p u c^{E 69}, d a p^{k 07309}$, UAS-Ras ${ }^{12}$, RFP-HP1, UAS-HP1-RNAi, UAS-upd1-RNAi, and UAS-cyclinERNAi.

Histology. Larval tissues were stained with standard immunohistochemical procedures using rabbit anti-Upd polyclonal antibody (1:500; a gift from D. Harrison), rabbit anti- $\beta$-galactosidase polyclonal antibody (Sigma, 1:500), mouse anti-phospho-JNK monoclonal antibody G9 (Cell Signaling, 1:100), mouse anti-Dacapo monoclonal antibody NP1 (Developmental Studies Hybridoma Bank, 1:6 for 7 days), mouse anti-BrdU monoclonal antibody BMG 6H8 (Roche, 1:100), rabbit anti-Histone $\mathrm{H} 3$ (tri methyl K9) polyclonal antibody (Abcam, 1:50), rabbit anti-RFP antibody (1:500), mouse anti-dmp53 monoclonal antibody p53 7A4 (Developmental Studies Hybridoma Bank, 1:500 for 4 days), mouse anti- $\gamma-\mathrm{H} 2 \mathrm{Av}$ monoclonal antibody UNC93-5.2.1 (ref. 56); Developmental Studies Hybridoma Bank, 1:200-400) or Alexa546-conjugated phalloidin (Molecular Probes, 1:50) and were mounted with DAPI-containing SlowFade Gold Antifade Reagent (Molecular Probes). For BrdU labelling, larvae were dissected in Schneider's medium and incubated in the same media with $10 \mathrm{mM}$ BrdU for $5 \mathrm{~min}$. After incubation with supplied buffer (Roche, 5-Bromo-2'-deoxyuridine labeling and detection kit I) containing $50 \mathrm{U} \mathrm{ml}^{-1} \mathrm{DNase} \mathrm{I}$, imaginal discs were stained with anti-BrdU monoclonal antibody BMG 6H8 (Roche, 1:100) and were mounted as descried above. Images were taken with a Zeiss LSM510, Zeiss LSM700 META (Zeiss) or Leica SP5 confocal microscope (Leica).

SA- $\beta$-gal staining. The SA- $\beta$-gal activity was visualized using the Senescent Cells Histochemical Staining Kit (CS0030, Sigma-Aldrich). Larvae were dissected in PBS and incubated in the $0.6 x$ Staining Mixture for $90 \mathrm{~h}$ at $37^{\circ} \mathrm{C}$. Images were taken with a stereomicroscope.

\section{References}

1. Bissell, M. J. \& Hines, W. C. Why don't we get more cancer? A proposed role of the microenvironment in restraining cancer progression. Nature Med 17, 320-329 (2011).

2. Jacks, T. \& Weinberg, R. A. Taking the study of cancer cell survival to a new dimension. Cell 111, 923-925 (2002).

3. Lowe, S. W., Cepero, E. \& Evan, G. Intrinsic tumour suppression. Nature 432, 307-315 (2004).

4. Rodier, F. \& Campisi, J. Four faces of cellular senescence. J. Cell Biol. 192, 547-556 (2011).

5. Collado, M., Blasco, M. A. \& Serrano, M. Cellular senescence in cancer and aging. Cell 130, 223-233 (2007).

6. Ohtani, N. \& Hara, E. Roles and mechanisms of cellular senescence in regulation of tissue homeostasis. Cancer Sci. 104, 525-530 (2013).

7. Bartkova, J. et al. DNA damage response as a candidate anti-cancer barrier in early human tumorigenesis. Nature 434, 864-870 (2005).

8. Michaloglou, C. et al. BRAFE600-associated senescence-like cell cycle arrest of human naevi. Nature 436, 720-724 (2005).

9. Braig, M. et al. Oncogene-induced senescence as an initial barrier in lymphoma development. Nature 436, 660-665 (2005).

10. Chan, H. M., Narita, M., Lowe, S. W. \& Livingston, D. M. The p400 E1Aassociated protein is a novel component of the p53--> p21 senescence pathway. Genes Dev. 19, 196-201 (2005).

11. Collado, M. et al. Tumour biology: senescence in premalignant tumours. Nature 436, 642 (2005).

12. Coppe, J. P., Desprez, P. Y., Krtolica, A. \& Campisi, J. The senescenceassociated secretory phenotype: the dark side of tumor suppression. Ann. Rev. Pathol 5, 99-118 (2010).

13. Young, A. R. \& Narita, M. SASP reflects senescence. EMBO Rep. 10, 228-230 (2009).

14. Davalos, A. R., Coppe, J. P., Campisi, J. \& Desprez, P. Y. Senescent cells as a source of inflammatory factors for tumor progression. Cancer Metastasis Rev. 29, 273-283 (2010).

15. Kuilman, T., Michaloglou, C., Mooi, W. J. \& Peeper, D. S. The essence of senescence. Genes Dev. 24, 2463-2479 (2010).

16. Kuilman, T. \& Peeper, D. S. Senescence-messaging secretome: SMS-ing cellular stress. Nat. Rev. Cancer 9, 81-94 (2009).

17. Coppe, J. P. et al. Senescence-associated secretory phenotypes reveal cellnonautonomous functions of oncogenic RAS and the p53 tumor suppressor. PLoS Biol. 6, 2853-2868 (2008). 
18. Bavik, C. et al. The gene expression program of prostate fibroblast senescence modulates neoplastic epithelial cell proliferation through paracrine mechanisms. Cancer Res. 66, 794-802 (2006).

19. Sparmann, A. \& Bar-Sagi, D. Ras-induced interleukin-8 expression plays a critical role in tumor growth and angiogenesis. Cancer Cell 6, 447-458 (2004).

20. Ancrile, B., Lim, K. H. \& Counter, C. M. Oncogenic Ras-induced secretion of IL6 is required for tumorigenesis. Genes Dev. 21, 1714-1719 (2007).

21. Park, E. J. et al. Dietary and genetic obesity promote liver inflammation and tumorigenesis by enhancing IL-6 and TNF expression. Cell 140, 197-208 (2010).

22. Yoshimoto, S. et al. Obesity-induced gut microbial metabolite promotes liver cancer through senescence secretome. Nature 499, 97-101 (2013).

23. Xu, T. \& Rubin, G. M. Analysis of genetic mosaics in developing and adult Drosophila tissues. Development 117, 1223-1237 (1993).

24. Ohsawa, S. et al. Mitochondrial defect drives non-autonomous tumour progression through Hippo signalling in Drosophila. Nature 490, 547-551 (2012).

25. Sakaue-Sawano, A. et al. Visualizing spatiotemporal dynamics of multicellular cell-cycle progression. Cell 132, 487-498 (2008).

26. Nakajima, Y., Kuranaga, E., Sugimura, K., Miyawaki, A. \& Miura, M. Nonautonomous apoptosis is triggered by local cell cycle progression during epithelial replacement in Drosophila. Mol. Cell Biol. 31, 2499-2512 (2011).

27. Lee, B. Y. et al. Senescence-associated beta-galactosidase is lysosomal beta-galactosidase. Aging Cell 5, 187-195 (2006).

28. Kosar, M. et al. Senescence-associated heterochromatin foci are dispensable for cellular senescence, occur in a cell type- and insult-dependent manner and follow expression of p16(ink4a). Cell Cycle 10, 457-468 (2011).

29. Di Micco, R. et al. Interplay between oncogene-induced DNA damage response and heterochromatin in senescence and cancer. Nat. Cell Biol. 13, 292-302 (2011).

30. Imai, Y. et al. Crosstalk between the Rb pathway and AKT signaling forms a quiescence-senescence switch. Cell Rep 7, 194-207 (2014).

31. Bartkova, J. et al. Oncogene-induced senescence is part of the tumorigenesis barrier imposed by DNA damage checkpoints. Nature 444, 633-637 (2006).

32. d'Adda di Fagagna, F. et al. A DNA damage checkpoint response in telomereinitiated senescence. Nature 426, 194-198 (2003).

33. Di Micco, R. et al. Oncogene-induced senescence is a DNA damage response triggered by DNA hyper-replication. Nature 444, 638-642 (2006).

34. Takai, H., Smogorzewska, A. \& de Lange, T. DNA damage foci at dysfunctional telomeres. Curr. Biol. 13, 1549-1556 (2003).

35. Rodier, F. et al. Persistent DNA damage signalling triggers senescenceassociated inflammatory cytokine secretion. Nat. Cell Biol. 11, 973-979 (2009).

36. Takahashi, A. et al. DNA damage signaling triggers degradation of histone methyltransferases through APC/C(Cdh1) in senescent cells. Mol. Cell 45, 123-131 (2012).

37. Martin-Blanco, E. et al. puckered encodes a phosphatase that mediates a feedback loop regulating JNK activity during dorsal closure in Drosophila. Genes Dev. 12, 557-570 (1998).

38. Igaki, T., Pagliarini, R. A. \& Xu, T. Loss of cell polarity drives tumor growth and invasion through JNK activation in Drosophila. Curr. Biol. 16, 1139-1146 (2006).

39. Sykiotis, G. P. \& Bohmann, D. Keap1/Nrf2 signaling regulates oxidative stress tolerance and lifespan in Drosophila. Dev. Cell 14, 76-85 (2008).

40. Igaki, T. et al. Eiger, a TNF superfamily ligand that triggers the Drosophila JNK pathway. EMBO J. 21, 3009-3018 (2002).

41. Uhlirova, M., Jasper, H. \& Bohmann, D. Non-cell-autonomous induction of tissue overgrowth by JNK/Ras cooperation in a Drosophila tumor model. Proc. Natl Acad. Sci. USA 102, 13123-13128 (2005).

42. Serrano, M., Lin, A. W., McCurrach, M. E., Beach, D. \& Lowe, S. W. Oncogenic ras provokes premature cell senescence associated with accumulation of p53 and p16INK4a. Cell 88, 593-602 (1997).

43. Kamijo, T. et al. Tumor suppression at the mouse INK4a locus mediated by the alternative reading frame product p19ARF. Cell 91, 649-659 (1997).

44. Kilbey, A., Terry, A., Cameron, E. R. \& Neil, J. C. Oncogene-induced senescence: an essential role for Runx. Cell Cycle 7, 2333-2340 (2008).

45. Shlevkov, E. \& Morata, G. A dp53/JNK-dependant feedback amplification loop is essential for the apoptotic response to stress in Drosophila. Cell Death Differ. 19, 451-460 (2012).
46. Owusu-Ansah, E., Yavari, A., Mandal, S. \& Banerjee, U. Distinct mitochondrial retrograde signals control the G1-S cell cycle checkpoint. Nature Genet. 40 , 356-361 (2008).

47. Uhlirova, M. \& Bohmann, D. JNK- and Fos-regulated Mmp1 expression cooperates with Ras to induce invasive tumors in Drosophila. EMBO J. 25, 5294-5304 (2006).

48. Pribluda, A. et al. A senescence-inflammatory switch from cancer-inhibitory to cancer-promoting mechanism. Cancer Cell 24, 242-256 (2013).

49. Pedersen, P. L. Tumor mitochondria and the bioenergetics of cancer cells. Prog Exp. Tumor Res. 22, 190-274 (1978).

50. Chien, Y. et al. Control of the senescence-associated secretory phenotype by NF- $\kappa \mathrm{B}$ promotes senescence and enhances chemosensitivity. Genes Dev. 25, 2125-2136 (2011).

51. Salminen, A., Kauppinen, A. \& Kaarniranta, K. Emerging role of NF-kappaB signaling in the induction of senescence-associated secretory phenotype (SASP). Cell Signal. 24, 835-845 (2012).

52. Harvey, K. F., Zhang, X. \& Thomas, D. M. The Hippo pathway and human cancer. Nat. Rev. Cancer. 13, 246-257 (2013).

53. Lu, L. et al. Hippo signaling is a potent in vivo growth and tumor suppressor pathway in the mammalian liver. Proc. Natl Acad. Sci. USA 107, 1437-1442 (2010).

54. Muñoz-Espín, D. et al. Programmed cell senescence during mammalian embryonic development. Cell 155, 1104-1118 (2013).

55. Lee, T. \& Luo, L. Mosaic analysis with a repressible cell marker for studies of gene function in neuronal morphogenesis. Neuron 22, 451-461 (1999).

56. Lake, C. M., Holsclaw, J. K., Bellendir, S. P., Sekelsky, J. \& Hawley, R. S. The development of a monoclonal antibody recognizing the Drosophila melanogaster phosphorylated histone $\mathrm{H} 2 \mathrm{~A}$ variant (gamma-H2AV). G3 (Bethesda, Md) 3, 1539-1543 (2013).

\section{Acknowledgements}

We thank A. Betsumiya, K. Nakagawa and T. Sawada for technical support; A.H.M. Alam for comments on the manuscript; D. Harrison for antibodies; T. Adachi-Yamada, U. Banerjee, D. Bohmann, Y. Hiromi, F. Missirlis, M. Miura, H. Sun, M. Uhlirova, T. Xu, the Bloomington Stock Center, the Vienna Drosophila RNAi Center, the National Institute of Genetics Stock Center and the Drosophila Genetic Resource Center for fly stocks. This work was supported in part by grants from the Ministry of Education, Culture, Sports, Science and Technology (MEXT) to S.O. and T.I., Grant-in-Aid for Scientific Research on Innovative Areas from the MEXT to S.O. and T.I., the Japan Society for the Promotion of Science for Young Scientists to S.O. and M.N., the Japan Science and Technology Agency to T.I., the Global COE program for Global Center for Education and Research in Integrative Membrane Biology to S.O. and T.I. R1 (1:250), mouse anti-phospho-or fly stocks., D. Bilder, Pernille Rorth antibody l antiboidy R1 (1:250), mouse anti-phospho-, Tomizawa Jun-ichi \& Keiko Fund of Molecular Biology Society of Japan for Young Scientist to S.O., the Novartis Foundation for the Promotion of Science to S.O, the Nakajima Foundation to S.O., the Inoue Science Research Award to S.O., the Takeda Science Foundation to S.O. and T.I., the Senri Life Science Foundation to S.O. and T.I., the Kao Function for Art and Science to S.O., the Shiseido Female Researcher Science grant to S.O., Uehara Memorial Foundation to S.O. and T.I., Suzuken Memorial Foundation to T.I., Inamori Foundation to T.I. and the Human Frontier Science Program Career Development Award to T.I.

\section{Author contributions}

M.N., S.O. and T.I. designed the research, M.N. and S.O. performed the experiments, M.N., S.O. and T.I. analysed the data, and M.N., S.O. and T.I. wrote the manuscript.

\section{Additional information}

Supplementary Information accompanies this paper at http://www.nature.com/ naturecommunications

Competing financial interests: The authors declare no competing financial interests

Reprints and permission information is available online at http://npg.nature.com/ reprintsandpermissions/

How to cite this article: Nakamura, M. et al. Mitochondrial defects trigger proliferation of neighbouring cells via a senescence-associated secretory phenotype in Drosophila. Nat. Commun. 5:5264 doi: 10.1038/ncomms6264 (2014). 